\title{
Efficient Resource Management for Multicast Ad Hoc Networks: Survey
}

\author{
Amit Chopra \\ PhD Research Scholar, CSE Deptt., MMEC, M. M. University, Ambala, India \\ E-mail: amit_chopra99@yahoo.com \\ Dr. Rajneesh Kumar \\ Professor, PhD, CSE Deptt., MMEC, M. M. University, Ambala, India \\ E-mail: rgujral77@yahoo.com
}

\begin{abstract}
Group communication over multicast ad hoc network suffers from insufficient utilization of limited resources, i.e. shared channel, battery, data processing capabilities and storage space etc. Multicast routing protocol should be able to manage all these resources because their consumption depends upon different factors, i.e. Unicast/Multicast network operations, dynamic topology due to mobility, control overhead due to scalability, packet loss and retransmission due to collision and congestion etc. All these factors may cause unnecessary network load, delay and unfair resource utilization. However, multicast ad hoc routing protocols are more efficient than Unicast routing protocols, but they also suffer from performance degradation factors discussed above. Researchers have developed various layer wise solutions for resource optimization. In this paper, we will explore the different schemes for fair utilization of network resources.
\end{abstract}

Index Terms-Multicast, Ad Hoc, Wireless, QoS, Resource Management, Bandwidth, Energy Efficient.

\section{INTRODUCTION}

\subsection{Multicast Ad Hoc Networks}

Multicast based communication is an important network service which sends the data from a source to multiple destinations simultaneously by creating copies only when the links to the destinations split. Multicast routing trees can be constructed to transmit the data from the source to all the destinations with a minimum multicast tree cost that is used to evaluate the utilization of network resources [1]. A multicast packet is transmitted to all members of its destination group with the same reliability as regular unicast packets. Multicasting can reduce the cost of communication, consumption of energy, bandwidth, Sender/Router processing and delivery delay [2]. Ad hoc networks support various applications for civilian and defense operations where set up and maintenance of the infrastructure based network may not be feasible. Efficient communication over ad hoc networks can be achieved using multicast routing protocols [3] but fair utilization of network resources is a major issue for such type of networks where network operations are simultaneously performed by the different layers. Multicast protocol dependency over multiple layers may degrade the performance of the entire network, if any intermediate layer exhausts maximum resources for data processing. So it is essential to optimize the operation of each layer for efficient utilization. Following are the operations supported by different layers: [31]

Table 1. Layer Wise Network Operation(s)

\begin{tabular}{|c|l|}
\hline Layer(s) & \multicolumn{1}{|c|}{ Operation(s) } \\
\hline $\begin{array}{c}\text { Application } \\
\text { Layer }\end{array}$ & $\begin{array}{l}\text { Scalable Encoding, Error Correction, } \\
\text { Adaptive Joint Source/Channel Coding, } \\
\text { Network Coding for Relay Networking }\end{array}$ \\
\hline Transport Layer & Error Correction \\
\hline Network Layer & Network Coding for Relay Networking \\
\hline MAC Layer & $\begin{array}{l}\text { Unicast packets processing, Polling, Error } \\
\text { Detection/Correction, Packet Retransmission }\end{array}$ \\
\hline Physical Layer & $\begin{array}{l}\text { Signal Processing, Space Time Coding, } \\
\text { Resource Allocation }\end{array}$ \\
\hline
\end{tabular}

Operations of all these layers can be optimized for efficient utilization of limited resources. In section-II, we will explore the contribution of different researchers in relevant areas.

\subsection{Resource Categorization}

Table 2. Resource Categorization[3][31]

\begin{tabular}{|c|l|l|l|}
\hline $\begin{array}{c}\text { Available } \\
\text { Resource(s) }\end{array}$ & $\begin{array}{c}\text { Resource } \\
\text { Consumption }\end{array}$ & Limitation(s) & $\begin{array}{c}\text { Requirement( } \\
\text { s) }\end{array}$ \\
\hline $\begin{array}{c}\text { Battery } \\
\text { Power }\end{array}$ & $\begin{array}{l}\text { Packet } \\
\text { Transmission, } \\
\text { Packet } \\
\text { Reception, Data } \\
\text { Processing }\end{array}$ & $\begin{array}{l}\text { Low battery } \\
\text { backup }\end{array}$ & $\begin{array}{l}\text { Energy } \\
\text { Efficiency }\end{array}$ \\
\hline $\begin{array}{c}\text { Shared } \\
\text { Channel }\end{array}$ & Communication & $\begin{array}{l}\text { Limited/Shared } \\
\text { bandwidth, } \\
\text { unfair channel } \\
\text { allocation, } \\
\text { Collision }\end{array}$ & $\begin{array}{l}\text { Fair Channel } \\
\text { Allocation, } \\
\text { Optimum } \\
\text { bandwidth } \\
\text { utilization }\end{array}$ \\
\hline $\begin{array}{c}\text { Central } \\
\text { Processing } \\
\text { Unit (CPU) }\end{array}$ & Data & $\begin{array}{l}\text { Low } \\
\text { Processing } \\
\text { Power }\end{array}$ & $\begin{array}{l}\text { Fair } \\
\text { Scheduling }\end{array}$ \\
\hline $\begin{array}{c}\text { Node's } \\
\text { Memory }\end{array}$ & Data Storage & Low Capacity & $\begin{array}{l}\text { Memory } \\
\text { Optimization }\end{array}$ \\
\hline
\end{tabular}




\subsection{Design goals of Multicast Routing Protocols for Ad Hoc Networks}

To develop a multicast routing protocol, there is need to focus on various constraints like of dynamic topology, limited power, shared bandwidth, network security and scalability. Ad hoc Multicast routing protocols should be robust, efficient and reliable. It should manage the control overhead, QoS, dependency over unicast protocol and limited resources. Following Fig.1 shows the classification of multicast routing protocols:

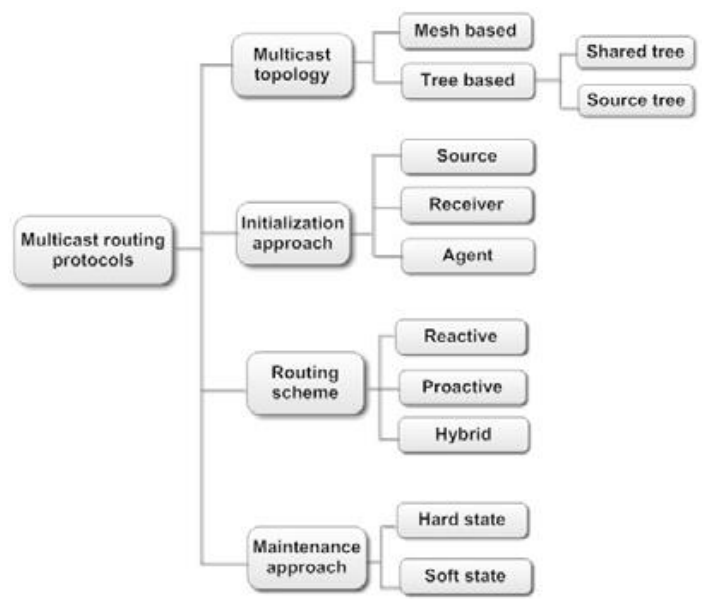

Fig.1. Classification of multicast routing protocols [32]

\subsection{Classification of Multicast Routing Protocols for Ad Hoc Network}

1. Multicast Topology: Ad hoc multicast routing protocols can be classified into two types: tree based and mesh based etc. In case of tree based, single link is established between source and destination pair where as in mesh based approach, there may be more then one links between source and destination pair. Tree based multicast protocols are most efficient but mesh based multicast protocols are more robust. Tree based multicast protocols can have the following types:

a. Source-Tree-based: in which each source maintains a separated tree that contains the source node as the root of the tree and all receivers lies under this node.

b. Shared-Tree-based: in which one tree is established in the entire network which includes all sources and receivers and links are routed to a main node that is referred core node [3][32].

2. Routing Initialization Approach: Multicast group formation can be initiated by source as well as by receivers. Routing initialization can be classified into three approaches:

a. Source-initiated: Source node initiates the group formation

b. Receiver-initiated: Receivers initiate the group formation

c. Hybrid approach: It uses the combination of both approaches

3. Routing Scheme: Routing scheme is classified into three approaches:

a. Proactive approach: Nodes maintain the routing tables for network topology

b. Reactive approach: Routes are established as per requirements

c. Hybrid approach: It uses the combination of both approaches [3][32].

4. Maintenance Approach: It is classified in to two different approaches:

a. Soft State: Multicasting Tree is updated periodically using control packets

b. Hard State: It is similar to Soft State except that it also uses a route repair method [3].

This article contains different sections i.e. Section-I introduce the basic requirements of resource management for multicast communication, Section-II explores the related research work in relevant fields. It provides brief overview of the various solutions developed for efficient utilization of resources. Section-III contains the open issues related to resource management and Section-IV concludes the outcome of this survey.

\section{RELATED WORK}

\subsection{Routing Solutions for fair Bandwidth utilization}

Salim Bitam et al. [6] proposed a QoS multicast routing protocol called MQBM for mobile ad hoc networks based upon the bee communication. It finds the routes between the source and the head of multicast group responsible to communicate the packet to the group members. A node can transmit the data only, if average end-to-end delay and the average bandwidth satisfy the QoS constraints. Simulation results show the efficiency and the performance of the proposed protocol after comparisons against MAODV in terms of the average end-to-end delay and the average bandwidth as QoS metrics.

Zheng Sihai et al. [7] proposed a QoS-based multicast routing protocol, called QMMRP. This protocol utilizes the entropy of node (a metric to measure the stability of ink) and bandwidth reservation policy to find a stable link with enough bandwidth. Simulation results show that the network load of QMMRP protocol is much higher than that of ODMRP protocol. On the basis of experiments, it can be concluded QMMRP performs better than the ODMRP.

G. Santhi et al. [9] proposed a Fuzzy cost based Multi constrained Quality of Service Routing (FCMQR) protocol that selects an optimal path by using independent QoS metrics such as bandwidth, end-to-end 
delay and intermediate hops etc. This is based on multi criterion objective fuzzy measure in which all the available resources of the path are converted into a single metric fuzzy cost. The path with the maximum lifetime (which is based on Mobility prediction) and minimum fuzzy cost will be considered to be the optimal one and used for multiple transmissions. Simulation results show that the FCMQR provides an accurate and efficient method to estimate and evaluate the QoS routing stability and cost in dynamic mobile networks.

G. Santhi et al. [11] described a method to select best paths from source to destination node in MANETs using fuzzy cost which is calculated on the basis of multiple independent QoS metrics such as bandwidth, end to end delay and the number of nodes to transfer data from the source to the destination. Authors compared their method with MAODV protocol and results show that proposed method performs well.

Y. Sun et al. [37] proposed an enhanced bandwidth efficient multicast routing for MANET. It optimizes multicast tree construction phase as well as a tree maintenance phase to reduce bandwidth consumption. Simulation results show its performance in terms of less control overhead with optimal bandwidth utilization.

\subsection{Energy Efficient Routing Solutions}

V. R. Budyal et al. [8] proposed a fuzzy agent based QoS multicast routing protocol for MANET by extending ODMRP. A group of agents are used to form a mesh based multicast network and a fuzzy logic system is used to select QoS nodes, which are selected on the basis of different parameters like speed, energy and delay etc. For packet routing, Qos mesh is used. The simulation results show the better packet delivery ratio with the increase in the number of nodes and mobility of the nodes.

Ting Lu et al. [10] proposed a genetic energy-efficient delay-constrained multicast routing algorithm to resolve the QoS multicast problem. It depends on bounded endto-end delay and minimum energy cost of the multicast tree. This algorithm is a source-based algorithm that calculates the route on the basis of energy consumption as well as on the end-to-end delay. Results show that the proposed algorithm is effective and efficient.

M. M. Qabajeh et al. [12] proposed a hierarchical scheme for multicast routing protocol with multiple QoS constrains over mobile ad hoc networks. This scheme is optimized to utilize the limited resources and reduces the overhead significantly. It searches for QoS paths from a single source to a set of destinations. Entire physical space is divided into equal size hexagonal cells and a leader and backup leader nodes is selected those are responsible to maintain network topology. Authors compared the purposed scheme with the other multicast routing protocol, called Demand Multicast Routing Protocol and results show that it performs well by reducing control over head with less packet drop ratio.

Salim Bitam et al. [13] proposed a novel bee colony optimization algorithm (BLA) for the QoS multicast routing problem (QoS-MRP) over vehicular ad hoc networks as NP-Complete problem with multiple constraints. BLA solved the QoS-MRP by achieving different constraints, i.e. cost, delay, jitter and minimum bandwidth. Results show the performance of this method by comparing with genetic algorithm (GA), bee algorithm (BA) and marriage in honey bee optimization (MBO) algorithm using same QoS constraints.

$\mathrm{Xu} \mathrm{Li} \mathrm{et} \mathrm{al.} \mathrm{[14]} \mathrm{proposed} \mathrm{a} \mathrm{Multicast} \mathrm{Ad} \mathrm{hoc} \mathrm{On-}$ demand Vector with Backup Branches (MAODV-BB) that is an enhancement of MAODV by merging the advantages of the tree structure and the mesh structure. It uses shorter tree branches and constructs a multicast tree with backup branches. Mathematical analysis and simulation results show that the MAODV-BB protocol can manage the quality of services even during heavy network load.

Ritesh K. Kalle et al. [15] proposed a energy efficient method for VoIP communication over IEEE-802.16m based networks by reducing overheads. During the data transmission phase, mobile station remains active and uses packet buffering. As per their analytical analysis, they categorized the power consumption in different modes i.e. idle mode, transmission mode and receiving mode and finally they examined the cycles in each mode. Simulation results show that power can be preserved in sleep mode by optimizing the MAC layer, by reducing overhead and delay etc.

Ya-li WANG et al. [16] proposed an ant colony-based multi-constrained QoS energy-saving routing algorithm (IAMQER) algorithm based on the analysis of local node information that considers various parameters related to nodes like queue length, number of forwarding data packets, residual energy etc. It can manage the throughput and the energy consumption simultaneously to improve the network performance in multi-constrained QoS routing. Simulation results show that IAMQER algorithm can handle the constraints like end-to-end delay and packet loss ratio and can find out the QoS routes and reduces average energy consumption and improves packet delivery ratio.

Jiwen Guo et al. [30] proposed a secure minimumenergy multicast (SMEM) algorithm to ensure multicast communication. In order to improve the stability of trust mechanism, the new trust values (calculated by the Bayesian theorem in CR networks) are modified by the iterative control criterion. Trust mechanism aims at guaranteeing the security of network environment, in which the trust information is encrypted to ensure the creditability of trust values. Results show that the time complexity of SMEM algorithm is polynomial.

S.M. Lakshmi et al. [35] developed an energy efficient routing method for multicast communication. Instead of using routing table, nodes use unique multicast id to transmit the data to a specific group. On demand routing path is built by calculating residual energy of intermediate multicast nodes and the distance between them. Simulation results show its performance in terms of minimum delay, extended network life time and enhanced Throughput. 


\subsection{MAC layer based solutions for efficient resource utilization}

Mui Van Nguyen et al. [17] proposed an optimal cognitive MAC protocol for MHAHNs under opportunistic spectrum access (OSA) approach in which channel contention resolution is done on the basis of interference-dependent random access addressing both social welfare maximization and energy efficiency. It offers contention control at MAC layer, interaction relationship with power control at the physical layer and also congestion control at the transport layer. They proposed an optimal cognitive MAC framework for NPhard problem. To solve this problem, authors introduced some auxiliary variables which are interpreted as interference weights and develop a distributed solution, which has been proved for global optimum to converge.

Pinyi Ren et al. [18] proposed MAC protocol based CAD (Channel-Aggregation Diversity) technology in which transmission time fairness constraints are introduced to transmit a particular number of packets at one time. Two joint power allocation schemes are introduced: First scheme is for high data transmission rate in which optimal allocation policy is used to solve Knapsack Problem. Dynamic programming is used to solve this. Second scheme is concerned with energy optimization which is resolved by fractional programming. Each node pair uses the RTS, CTS and RES for information exchange. If sender observes that channel is free than it send RTS to receiver. At receiver end, RTS is processed and CTS is sent to the sender. Finally, Sender sends RES in acknowledgement and starts the data transmission as per the channel allocation policy. Simulation results sow its performance in terms of energy efficiency and throughput.

M. Van Nguyen et al. [19] focused on the probabilistic channel contention resolution problem for net revenue maximization in multi-hop wireless ad-hoc networks (MHAHNs) under collision-rate-constrained opportunistic spectrum access (OSA) approach. Specifically, They considered the interference-dependent contention model, in which secondary users (SUs) must coordinate to each other to simultaneously balance between interference and collision, leading a more efficient MAC protocol than the location-dependent one proposed in the literature. By introducing some auxiliary variables and noisy channel estimations, they developed a novel heuristic cross-layer cognitive MAC protocol (HCC-MAC) to solve formulated MAC optimization problem which is shown non-convex and inseparable. Proposed protocol can achieve near-optimal throughput in a distributed manner without control overhead. Analytical analysis shows that HCC-MAC can outperform the existing MAC protocols under OSA paradigm.

Di Marco et al. [20] introduced the study of routing metrics and interaction of routing with MAC. Different concepts of cross layer interactions are introduced. MAC layer parameters as well as network performance both are affected by Contention levels. Some reliability constraints are enforced to increase the lifetime of network and also used to balance the network load. Proposed solutions are compared with routing approach based on backpressure mechanism and results show the efficiency of proposed solution.

Farooq M.O et al. [21] presented a bandwidth estimation-based admission control and routing protocol, called BEAR for IEEE 802.15.4 - based networks. The purpose of BEAR is to support real-time multimedia flows in IEEE 802.15.4 - based networks by satisfying their end-to-end bandwidth requirements. It combines a bandwidth estimation approach, admission control, and routing. It uses a measurement-based bandwidth estimation algorithm that combines nodes' transmission rate, and actual MAC layer overhead to estimate the available bandwidth. The available bandwidth information is used as input to the admission control protocol, and it supports algorithms to estimate a flow's contention, intra-flow contention, and additional MAC layer overhead with an increased data load inside a network. Available bandwidth information can be used as a routing metric to select forwarding path(s) that can offer better end-to-end available bandwidth. Proposed framework can be used to support real-time multimedia applications for IEEE 802.155.4 based networks.

Jackson.C.A et al. [22] proposed a hierarchical and heterogeneous multichannel ad hoc network. The channels employed by this network are non overlapping, and each channel differs significantly in its characteristics, such as achievable data rate, communication range, and traffic load. Every terminal is connected with the frequency-agile radio which can change its transmission rate and carrier frequency. These radios communicate using contention-based access and are permitted to utilize multiple channels. A subset of terminals forms a backbone network, which is equipped with a second radio tuned to a traffic channel employing schedule-based access. They considered various hierarchical and heterogeneous multichannel ad hoc network topologies and investigated the backbone networks that increase network-layer performance over scenarios in which too few or too many terminals are selected to form the backbone network.

Zheng $\mathrm{Li}$ et al. [23] proposed a novel multiplerecipient based on cooperative MAC layer protocol for wireless ad hoc networks. The sender selects a recipient among multiple candidate recipients. Candidate helpers employ a preliminary helper contention scheme and an optimal helper contention scheme to select the optimal helper according to their cooperation gain. Besides, the sender can send a data packet at the highest data rate that it can support according to the busy tones in the preliminary helper contention process, guaranteeing both the channel utilization and the link reliability. Once the recipient receives the sender's data packet through the optimal helper, it can also send its data packet to the sender through the same optimal helper. Furthermore, the optimal helper can piggyback its data packet to the sender or the recipient, reducing the reservation overhead and the possibility of collisions during channel reservation. 
Simulation results show the performance of proposed protocol compared with CoopMACA and 2rcMAC using different parameters such as throughput and average packet delay.

Haitao Zhao et al. [24] proposed a capacity-aware statistical QoS routing scheme which is used to realize statistical Quality of service guarantees for IEEE standard 802.11 wireless networks under a particular medium or for high traffic load. This proposed scheme estimates the probability of packet collision through MAC layer retransmission and the statistical traffic load dynamically, thereby achieving the capacity prediction of wireless channel based on statistical probability and service process. Furthermore, a cross layering design approach is used, in which proposed scheme offers a stochastic QoS route optimization and the traffic flow admission control in terms of capacity-aware criteria and the location-based route discovery to obtain effective resource utilization and packet forwarding. Simulation results show that this scheme can detect the state of wireless link well. Also, it decreases end-to-end delay and improves successful packet delivery percentage without increasing routing overhead over multi-hop wireless networks. The simulation shows the effectiveness of the proposed scheme over the existing location-based QoS optimization delivery algorithm in terms of retransmission count, successful delivery rate and end to end delay on the condition of time-varying multi-hop wireless links.

Alessandro Delfino et al. [25] presented the opinion for design and implementation of medium access control in which they use ECMA-392 standard. They basically focused on the centralized cognitive network, in which network is considered which uses out-of-band cognitive control channel (CCC). This CCC is used to manage different types of operations of the network. Proposed work shows that the capability of the network should be reconstructed or reconfigured when CCC is jammed by any primary user. They designed a prototype for average network reconfiguration delay which is used to operate in 375-445 MHz frequency bands for public safety ad hoc networks.

$\mathrm{Ze} \mathrm{Li}$ et al. [26] proposed the enhancement in hybrid approach by using Quality of Service support capability. This new protocol named as QoS Oriented Distributed Routing Protocol (QOD) which used the advantages of anycast and fewer transmission hops. There are five algorithms for QOD- First algorithm is for QoS guaranteed neighbor selection which is used to overcome the transmission delay requirement. Second algorithm is to reduce transmission delay is distributed packet scheduling algorithm. Third algorithm is to reduce time of transmission is mobility based segment resizing algorithm which can adjust its segment size according to the mobility of nodes. Fourth algorithm is used to increase throughput is traffic redundant elimination algorithm and Fifth algorithm is used to remove the redundancy in data is Data Redundancy eliminationbased transmission algorithm. Simulation results shows the performance of QOD which can achieve more scalability, reduce the contention and high mobilityresilience.

K.H. Almotairi et.al [27] presented a MAC protocol based on the distributed multi-channel that can utilize hopping sequences with multiple radio interfaces for transmission and reception. They proposed an analytical model and simulation results validate this model and show the performance in terms of Throughput.

Bourdelles, M et.al [28] proposed a CPU resources control module to be included in a real-time embedded system, and using platform resources tuning capabilities such as Dynamic Voltage and Frequency Scaling (DVFS). This control module has been designed to be generic enough to be adapted to any system, but precise enough to take into account real-time constraints of such systems. The solution proposed does not need any knowledge and pre-execution of the business code of the active components of the system to be monitored. The solution is demonstrated on the Medium Access Control (MAC) of a radio protocol implemented on a free scale IMX6 board with power gains of more than $40 \%$ with frequency scale variations from $800 \mathrm{MHz}$ to $400 \mathrm{MHz}$.

Fumihisa Yamanobe et al. [29] proposed a mobile agent based solution for inter-path interference using multipath under mobility constraints. It can estimate the routes on the basis of the shortest path method and as per route request, it can build single or multipath and it can also perform the load balancing by reducing the control overhead. To handle the broken links, it does not flood the control packets, broken link information is delivered by unicast method instead of broadcast and it reduces the interference over a network. It can also regulate the TCP connections to avoid the excessive network load as well as the interference over the channel.

Xueyuan $\mathrm{Su}$ et al. [36] focused on the bandwidth allocation problem and explored the network and MAC layer impact over the existing bandwidth allocation methods, i.e. Interference model, Rate allocation scheme and contention based algorithms. The study shows that cross layer solutions can be used to regulate the data flow rate across both layers.

\section{PROBLEM FORMULATION}

\subsection{Multicast Routing Constraints}

MANET based applications are widely used in the remote areas where rapid deployment and dynamic reconfiguration is necessary and wired network support is unavailable. These include military battlefields, emergency search, rescue sites, classrooms and conventions, where communication can be initialized using audio/video streams with the help of wireless nodes. In case of voice communication over MANETs, we transmit audio stream having a high data rate and it reduces the voice quality due to the usage of low-power wireless links. Transmission of concurrent voice streams can reduce the efficiency of the entire network. Voice stream multicast can play an important role in case of 
emergency situations. In case of video communication over MANETs, it requires efficient bandwidth and real time support but due to the limited resources and error prone network environment, it becomes challenging to manage the quality of video transmission as well as the network bandwidth.

Multicast routing plays a significant role in MANETs and multicasting can improve the efficiency of the wireless links but it is difficult and challenging to design an effective and reliable multicast routing protocol for MANETs. In recent years, various multicast routing protocols have been proposed for MANETs to support different services. Multicast communication over ad hoc networks has its own limitations but it also suffers from common issues, i.e. dynamic topology, performance of routing protocol, inefficient resource management, security, scalability, QoS, QoE etc. If we use the multicasting over the MANETs then there is need to explore and sort out these issues for the multicast mobile ad hoc networks. Traditional wired Multicast protocols are not suitable for ad hoc networks because the routing tree becomes unstable due to the dynamic network environment. [3].

Quality of communication is affected by noise/interference and channel fading. Multicast routing protocol must be able to regulate the flow of control packets over a network, in order to maintain the channel capacity [33]. There may be packet drop due to the dynamic change in the network topology that can reduce the network performance. So multicast routing should be able to operate in different situations such as mobile environment, heavy traffic load, scalability in order to achieve high packet delivery [3].

\subsection{Resource management issues}

Efficient resource utilization is a critical issue for Multicast Ad hoc networks, which deals with consumption of various network resources discussed below:

a. Node Energy: Every node has limited battery life and it is very difficult to manage the energy level of individual node because each node may participate in different network operations and its energy level is reduced as per the performed task [3][34].

b. Shared Channel Bandwidth allocation: Ad hoc networks utilize shared channel and fair channel allocation becomes a major issue [30].

c. Node Level Buffer management: Due to packet loss/link loss, retransmission phase is executed by routing protocol, thus may result in buffer overflow, network congestion/contention etc [3].

\subsection{Resource utilization dependencies}

a. Network size/topology: Due to the dynamic network topology and network size, route discovery and route maintenance phase are performed by protocols and thus results in degradation of network performance.

b. Node density/mobility: Node Mobility and scalability can degrade the QoS of the entire network and it is difficult to maintain network performance under these constraints. [31]

c. Traffic type/load: Real time applications consume network resources at large scale. In insufficient bandwidth allocation may result in delay and can degrade the quality of experience of end users.

\subsection{Behavioral Impact of different layers over network resource consumption}

a. Multicast Routing Layer: Routing layer performs different network operations such as route discovery, route maintenance and session management etc. Routing layer operations may suffer from the dynamic behavior of the ad hoc networks and thus may result in the link errors and frequent update of routing information. Due to excessive route maintenance, node's life time can be exhausted [3].

b. MAC Layer: It communicates with two different layers, i.e. routing layer and physical layer. It also manages contention over wireless channel [3]. MAC protocols directly affect the channel allocation, bandwidth utilization, contention and packet collision etc. Collision at large scale can produce the delay and contention over network and QoS may be degraded. If MAC protocols fail to manage fair channel allocation, it can also degrade the performance of ad hoc networks in a multi user environment.

\section{CONCLUSION}

In this paper, we explored the issues and solutions related to resource management for multicast ad hoc networks. Routing layer based solutions include MQBM multicast routing protocol, which can maintain end-toend delay and the average bandwidth as QoS metrics, QMMRP supports QoS as compared to ODMRP, fuzzy logic based agents, path selection and QoS aware routing both are capable to adopt node's mobility and scalability and also improve the packet delivery ratio, delayconstrained multicast routing algorithm and buffer management scheme can minimize the delay in order to preserve the energy, hierarchical based multicasting can reduce packet loss, delay and can improve network efficiency, Ant colony based multicasting and MAODVBackup Branch both can fulfill QoS constraints.

MAC layer based solutions include methods for channel aggregation Diversity, power and congestion control, channel allocation policy, MAC optimization, bandwidth estimation-based admission control, heterogeneous multichannel for backbone networks, capacity-aware statistical QoS routing, QoS Oriented Distributed Routing, distributed multi-channel support, 
CPU resources management for MAC protocol, interference management and network load balancing etc. Finally we can conclude that there is need to develop a cross layer solution which can encounter common issues related to each layer because these can have an impact over the operations of other layer. In future, this study will be used for performance analysis of multicast protocols under the constrained of limited resources.

\section{REFERENCES}

[1] Hui Cheng et al., "Hyper-mutation based Genetic Algorithms for Dynamic Multicast Routing Problem in Mobile Ad Hoc Networks", 11th International Conference on Trust, Security and Privacy in Computing and Communications-2012 IEEE, pp. 1586-1592.

[2] N. Bhalaji et al., "Performance Comparison of Multicast Routing Protocols under Variable Bit Rate Scenario for Mobile Adhoc Networks", Recent Trends in Network Security and Applications Communications in Computer and Information Science vol. 89, 2010, Springer-2010, pp. 114-122.

[3] C. Siva Ram Murthy, B.S. Manoj, "Ad Hoc Wireless Networks”, 14 impression-Pearson-2012, Chapter (5-11), pp. 191-641.

[4] Priyanka Das, Veena Gulhane, "Distributed Energy Adaptive Location-Based Cooperative MAC Protocol for Prolonging the Network Lifetime of MANET", ACCT, IEEE, 2015, pp.298-303.

[5] Lav Gupta, R. Jain, G. Vaszkun, "Survey of Important Issues in UAV Communication Networks", Communications Surveys \& Tutorials, IEEE-2015, pp.132.

[6] Salim Bitam et al., "MQBM: an autonomic QoS multicast routing protocol for mobile ad hoc networks", ICC-IEEE2012, pp. 5488-5492.

[7] Zheng Sihai et al., "QoS-Based Multicast Routing Protocol in MANET", International Conference on Industrial Control and Electronics Engineering, IEEE2012, pp. 262-265.

[8] V. R. Budyal et al., "Fuzzy Agent based Quality of Service Multicast Routing in Mobile Ad hoc Network", International Conference on Advances in Mobile Network, Communication and Its Applications, IEEE-2012, pp. 9598.

[9] G. Santhi et al., "Fuzzy-Cost based Multicast QoS Routing with Mobility prediction in MANETs", ICAESM -IEEE-2012, pp. 556 - 562.

[10] Ting Lu, Jie Zhu, "Genetic Algorithm for EnergyEfficient QoS Multicast Routing", IEEE COMMUNICATIONS LETTERS, vol. 17(1), IEEE-2013, pp. 31-34.

[11] G. Santhi et al. "Fuzzy Cost Based Multicast Routing for Mobile Ad-Hoc Networks with Improved QoS", Advances in Digital Image Processing and Information Technology Communications in Computer and Information Science, vol. 205, 2011, Springer-2011, pp. 429-437.

[12] M. M. Qabajeh et al., "A Tree-based QoS Multicast Routing Protocol for MANETs", ICOM-Malaysia, IEEE2011, pp. 1-6.

[13] Salim Bitam, Abdelhamid Mellouk, "Bee life-based multi constraints multicast routing optimization for vehicular ad hoc networks", Journal of Network and Computer Applications, Vol. 36 (3), May 2013, pp. 981-991, Elsevier.
[14] Xu Li et.al, "Optimized multicast routing algorithm based on tree structure in MANETs", Communications, China, Vol. 11 (2), 2014, IEEE, pp. 31 - 34.

[15] Ritesh K. Kalle, Maruti Gupta, Aran Bergman, EladLevy, ShantidevMohanty, Muthaiah Venkatachalam, Debabrata Das, "Advanced Mechanisms for Sleep Mode Optimization of VoIP Traffic over IEEE $802.16 \mathrm{~m} "$, IEEE, Globecom 2010, pp 1-6.

[16] Ya-li WANG et.al, "Improved ant colony-based multiconstrained QoS energy-saving routing and throughput optimization in wireless Ad-hoc networks", The Journal of China Universities of Posts and Telecommunications, Vol. 21 (1), Elsevier, 2014, Pages 43-53, 59.

[17] Nguyen Mui Van, Hee Kyung., Yongin, Seon Hong Choong, "Interference-dependent contention control in multi hop wireless ad-hoc networks: An optimal cognitive MAC protocol", ICC, IEEE-2013, pp.6163 - 6167

[18] Pinyi Ren, Xi'an Jiaotong, Xi'an, China; Yichen Wang; Qinghe Du, "CAD-MAC: A Channel-Aggregation Diversity Based MAC Protocol for Spectrum and Energy Efficient Cognitive Ad Hoc Networks", IEEE Journal Vol.32 (2), FEB. 2014, 2-13, pp.237 - 250.

[19] M. Van Nguyen, K. Hee, Yongin, C. Seon Hong ; L. B. Le," Cross-layer cognitive MAC design for multi-hop wireless ad-hoc networks with stochastic primary protection", WCNC, 2013 IEEE, pp.1802 - 1807

[20] Di Marco, Stockholm, Fischione, C.; Athanasiou, G.; Mekikis, P.-V., "MAC-aware routing metrics for low power and lossy networks", INFOCOM, 2013 Proceedings IEEE, pp. $13-14$

[21] Farooq, M.O.; Inst. of Telematics, Univ. of Luebeck, Luebeck, Germany; Kunz, T., "Proactive Bandwidth Estimation for IEEE 802.15.4-Based Networks ", VTC Spring-IEEE-2013, pp.1-5

[22] Jackson, C.A., Clemson, SC. Russell, H.B. Wolf, B.J, Martin J, "Investigation of MAC for a Hierarchical and Heterogeneous Multichannel Ad Hoc Network", MILCOM 2013 - IEEE, pp. 188 - 193

[23] Zheng Li., Kai Liu, "A multiple-recipient-based cooperative MAC protocol for wireless ad hoc networks", MAPE, 2013-IEEE, pp.59-64

[24] Haitao Zhao, Hongbo Zhu, Dapeng Li, Nanjie Liu, "Quality-Driven Capacity-Aware Resources Optimization Design for Ubiquitous Wireless Networks", Wireless Pers Commun 2014, Springer, pp.329-344

[25] Alessandro Delfino, Leonardo Goratti, Raimondo Giuliani, Franco Oliveri, Gianmarco Baldini, "A Software Radio Implementation of Centralized MAC Protocol for Cognitive Radio Networks", Wireless Personal Communication -2013, Springer, pp.1147-1175

[26] Ze Li, Haiying Shen, "A QoS-Oriented Distributed Routing Protocol for Hybrid Wireless Networks", IEEE Transactions on mobile computing, Vol. 13, (3), IEEE2014, pp.693-708

[27] Almotairi, K.H., UmmAl-Qura Univ., Makkah, Shen, X.S.,"A Distributed Multi-Channel MAC Protocol for Ad Hoc Wireless Networks ", Mobile Computing, IEEE Transactions, Vol.14 (1), IEEE-2015, pp.1 - 13

[28] Bourdelles, M., Marechal, J., "Power consumption optimization for real-time applications: Results on a MAC application implementation", INFOCOM WKSHPS, IEEE-2014, pp.753 - 758

[29] Fumihisa Yamanobe, Shigeki Shiokawa, "Multi-path Routing Considering Inter-path Interference Using a Mobile Agent In the Ad-hoc Networks", APCC, IEEE 2014, pp.15-19

[30] Jiwen Guo et al., "Secure Minimum-Energy Multicast 
Tree Based on Trust Mechanism for Cognitive Radio Networks", Wireless Personal Communications November 2012, vol. 67 (2), Springer-2012, pp. 415-433.

[31] Jean-Marie Vella, Saviour Zammit, "A Survey of Multicasting over Wireless Access Networks", IEEE Communications Surveys \& Tutorials, Vol. 15 (2), IEEE2013, pp.718-753.

[32] Ahmed. M. Abdel Mo'men et al., "A Survey on Security Enhanced Multicast Routing Protocols in Mobile Ad Hoc Networks", HONET- IEEE-2010, pp. 262-268.

[33] Osamah S. Badarneh andMichel Kadoch, "Multicast Routing Protocols inMobile Ad Hoc Networks: A Comparative Survey and Taxonomy", EURASIP Journal on Wireless Communications and Networking, EURASIP-2009, pp.1-42.

[34] Kartik Chawda, Deepmala Gorana, "A survey of Energy Efficient Routing Protocol in MANET", ICECS, IEEE2015, pp.953-957

[35] S.M. Lakshmi, K.T. Sikamani, "Energy Aware Multicast Opportunistic Routing Protocol (Eamor) To Optimize Lifetime of Manet", ICCTET, IEEE-2013, pp.210-212.

[36] Xueyuan Su, Sammy Chan, "Bandwidth Allocation in Wireless Ad Hoc Networks: Challenges and Prospects", IEEE Communications Magazine, pp.80-85.

[37] Y. Sun, R. Sun, F. Jiang, C. Phillips, "A new bandwidth- efficient multicast routing scheme for mobile Ad hoc Networks", WTS, IEEE-2015, pp.1-7.

\section{Authors' Profiles}

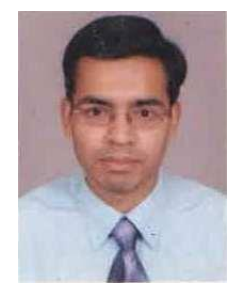

Amit Chopra received his master's degree in Computer Science and Engineering from M. M. Engineering College, M. M. University, Ambala, India and pursuing $\mathrm{PhD}$ (CSE) from same University and his research interest includes Wireless Ad Hoc/Sensor Networks, Multimedia Communication, Cryptography and Network

Security etc.

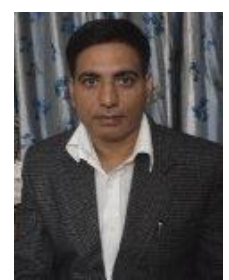

Dr. Rajneesh Kumar is working as Professor at M. M. Engineering College, M. M. University, Ambala, India and his research interests are Wireless Networking and communications and he has published several papers in International Journals and conferences.

How to cite this paper: Amit Chopra, Rajneesh Kumar,"Efficient Resource Management for Multicast Ad Hoc Networks: Survey", International Journal of Computer Network and Information Security(IJCNIS), Vol.8, No.9, pp.4855, 2016.DOI: 10.5815/ijcnis.2016.09.07 